\title{
PERFECT CODES ON THE TOWERS OF HANOI GRAPH
}

\author{
ChI-Kwong Li ANd INGRId NeLSON
}

\begin{abstract}
We characterise all the perfect $k$-error correcting codes that can be defined on the graph associated with the Towers of Hanoi puzzle. In particular, a short proof for the existence of 1-error correcting code on such a graph is given.
\end{abstract}

\section{INTRODUCTION}

In the study of recurrence relations, one common example is the following combinatorial game known as the Towers of Hanoi puzzle.

Initially, there are 3 pegs and $n$ circular disks of increasing size on one peg with the largest disk on the bottom. These disks are to be transferred one at a time onto another of the pegs with the provision that one is never allowed to place a larger disk on top of a smaller one. The problem is to determine the number of moves necessary for the transfer.

For convenience, we call the three pegs $P_{0}, P_{1}$, and $P_{2}$, and label the disk as $D_{1}, \ldots, D_{n}$, where $D_{1}$ has the smallest radius. Define a legal configuration of the disks on the three pegs to be an arrangement of the disks on the pegs so that no larger disk is on the top of a smaller one. Then one easily checks that there is a one-one correspondence between all legal configurations with the space $\mathbb{Z}_{3}^{n}$ of ternary sequences of length $n$, such that a given $\mathbf{x}=x_{1} \cdots x_{n} \in \mathbb{Z}_{3}^{n}$ corresponds to the configuration with $D_{i}$ lying on $P_{j}$ if $x_{i}=j$. For example, 101 corresponds to the configuration that $D_{1}$ and $D_{3}$ lie on $P_{1}$, and $D_{2}$ lies on $P_{0}$ (see Figure 1).

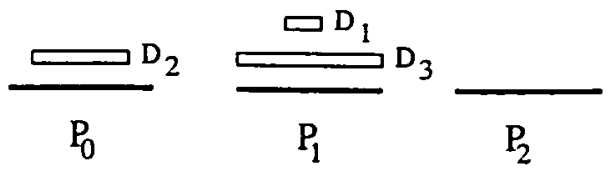

Figure 1

We shall call the legal configuration corresponding to $\mathbf{x} \in \mathbb{Z}_{3}^{n}$ the $\mathbf{x}$-configuration. The sequences with all entries equal to the same $i \in\{0,1,2\}$ are called the perfect states corresponding to the configurations with all disks lying on the same peg.

One can construct a graph with all $\mathbb{Z}_{3}^{n}$ as the vertex set, where two vertices $\mathbf{x}$ and $y$ are connected by an edge if there is a legal move in the Towers of Hanoi puzzle that

Received 26th August, 1997

Copyright Clearance Centre, Inc. Serial-fee code: 0004-9729/98 \$A2.00+0.00. 
transforms the $\mathbf{x}$-configuration to the $\mathbf{y}$-configuration. This graph is called the Towers of Hanoi graph, denoted by $H_{n}$, and first appeared in [6]. We depict $H_{1}$ and $H_{2}$ in Figure 2.
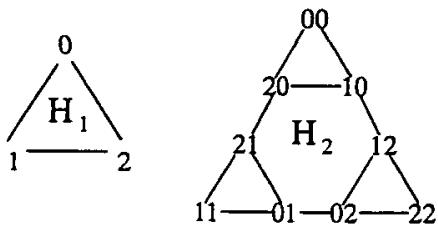

Figure 2

The Towers of Hanoi puzzle, its graph, and their generalisations have generated much interesting research (for example, see [5]). In fact, the graph $H_{n}$ can be constructed from $H_{n-1}$ by the following algorithm:

Step 1. Let $\widetilde{H}_{n-1}$ be the mirror image of $H_{n-1}$ about a vertical line passing through the top perfect state.

Step 2. Construct $H_{n-1}^{(i)}$ by appending $i$ to the end of each vertex of $\widetilde{H}_{n-1}$ to form a sequence of length $n$ for $i=0,1,2$.

Step 3. Put $H_{n-1}^{(0)}$ in the top, rotate $H_{n-1}^{(1)}$ by 120 degrees clockwise and put it in the left bottom corner, rotate $H_{n-1}^{(2)}$ by 120 degrees counterclockwise and put it in the right bottom corner.

Step 4. Connect the vertex $0 \cdots 01$ in $H_{n-1}^{(1)}$ with the vertex $0 \cdots 02$ in $H_{n-1}^{(2)}$, connect the vertex $1 \cdots 10$ in $H_{n-1}^{(0)}$ with the vertex $1 \cdots 12$ in $H_{n-1}^{(2)}$, connect the vertex $2 \cdots 20$ in $H_{n-1}^{(0)}$ with the vertex $2 \cdots 21$ in $H_{n-1}^{(1)}$.

One easily sees that this algorithm will generate all $x \in \mathbb{Z}_{3}^{n}$ as vertices, and all the legal moves in the Towers of Hanoi puzzle as edges. We give the graphical representation of the situation in Figure 3.

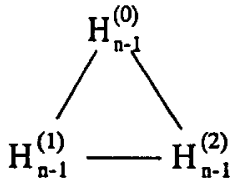

Figure 3

Define the distance $d(\mathbf{x}, \mathbf{y})$ between two vertices $\mathbf{x}$ and $\mathbf{y}$ to be the length of the shortest path joining the two vertices. Clearly, $d(\mathbf{x}, \mathbf{y})$ corresponds to the minimum number of legal moves needed in the Towers of Hanoi puzzle to transform the $\mathrm{x}$-configuration to the $\mathbf{y}$-configuration. For example (for example, see [2]), the distance between 2 perfect states in $H_{n}$ equals $2^{n}-1$, which is the maximum distance between any two vertices in $H_{n}$. The distance function $d$ defines a metric on $\mathbb{Z}_{3}^{n}$, and for any nonnegative integer $k$, one may define the radius- $k$ ball centred at $\mathrm{x} \in \mathbb{Z}_{3}^{n}$ to be the set

$$
B(\mathbf{x}, k)=\left\{\mathbf{y} \in \mathbb{Z}_{3}^{n}: d(\mathbf{x}, \mathbf{y}) \leqslant k\right\} .
$$

In the study of coding theory (see [4] for general background), one would like to 
partition $\mathbb{Z}_{3}^{n}$ as a disjoint union of $B\left(\mathbf{x}_{1}, k\right), \ldots, B\left(\mathbf{x}_{M}, k\right)$ for a suitable choice of $\mathbf{C}_{n}(k)=$ $\left\{\mathbf{x}_{1}, \ldots, \mathbf{x}_{M}\right\} \subseteq \mathbb{Z}_{3}^{n}$. If such a partition exists, the set $\mathbf{C}_{n}(k)$ will be called a perfect $k$-error correcting ternary code on $H_{n}$.

The purpose of this note is to determine those $(n, k)$ pairs for which a perfect $k$-error correcting code exists on $H_{n}$, and characterise $\mathbf{C}_{n}(k)$ if it exists. In particular, we shall give a short proof for the existence of a perfect 1-error correcting code on $H_{n}$ (see [2] for the original proof).

It is worthwhile to point out that the existence of the $k$-error correcting codes on $\mathbb{Z}_{3}^{n}$ depends heavily on the structure of the radius $k$-balls. If a different metric is used, then the structure of the radius $k$-balls will be completely changed, and hence the corresponding coding theory problem will change accordingly. For instance, if the widely used Hamming metric $\tilde{d}(\mathbf{x}, \mathbf{y})=$ the number of no zero entries in the vector $(\mathbf{x}-\mathbf{y})$, then a perfect 1-error ternary correcting code rarely exists (see [4]).

Note also that other coding theory problems have been studied using the Towers of Hanoi graph [3].

\section{Results AND Proofs}

We begin with a short proof of the fact that perfect 1-error correcting codes always exists on $H_{n}$. See [2] for the original proof.

THEOREM 2.1. Let $n$ be a positive integer. Then the collection $\mathbf{C}_{n}(1)$ of ternary sequences of length $n$ with an even number of terms equal to 1 and an even number of terms equal to 2 is a perfect 1-error correcting code on $H_{n}$. Moreover, we have

$$
\left|\mathbf{C}_{n}(1)\right|= \begin{cases}\left(3^{n}+3\right) / 4 & \text { if } n \text { is even } \\ \left(3^{n}+1\right) / 4 & \text { if } n \text { is odd }\end{cases}
$$

Proof: We need to show that every $\mathbf{y} \in \mathbb{Z}_{3}^{n}$ lies in one and only one $B(\mathbf{x}, 1)$ with $\mathbf{x} \in \mathbf{C}_{n}(1)$. Denote by $n_{\boldsymbol{i}}$ the number of terms in $\mathbf{y}$ that equal $i$ for $i=0,1,2$.

First, suppose $\mathbf{y} \in \mathbf{C}_{n}(1)$, that is, $n_{1}$ and $n_{2}$ are even. Then $\mathbf{y} \in B(\mathbf{y}, 1)$, and it is clear that $\mathbf{y}$ cannot be transformed to another $\mathbf{x} \in \mathbf{C}_{n}(1)$ by just one legal move. Hence, $\mathbf{y}$ lies in $B(\mathbf{y}, 1)$ but not in $B(\mathbf{x}, 1)$ for any other $\mathbf{x} \in \mathbf{C}_{n}(1)$. Next, suppose $\mathbf{y} \notin \mathbf{C}_{n}(1)$. Then either

(i) both $n_{1}$ and $n_{2}$ are odd, or

(ii) exactly one of $n_{1}$ or $n_{2}$ is odd.

If (i) holds, then one can consider the $y$-configuration and transfer the smallest disk on $P_{1}$ and $P_{2}$ from one peg to the other peg. Clearly, the resulting configuration corresponds to a ternary sequence $\mathbf{x} \in \mathrm{C}_{n}(1)$, and the proposed move is the only single legal move on the $y$-configuration that will lead to a configuration corresponding to a sequence in $\mathbf{C}_{n}(1)$. Thus $\mathbf{y} \in B(\mathbf{x}, 1)$, but is not in any other unit ball $B(\mathbf{z}, 1)$ with $\mathbf{z} \in \mathbf{C}_{n}(1)$. 
Suppose (ii) holds, and suppose $n_{i}$ is odd with $i \in\{1,2\}$. Then one can consider the y-configuration and transfer the smallest disk on $P_{0}$ and $P_{i}$ from one peg to the other peg. Clearly, the resulting configuration corresponds to a ternary sequence $\mathbf{x} \in \mathbf{C}_{n}(1)$, and the proposed move is the only single legal move on the $y$-configuration that will lead to a configuration corresponding to a ternary sequence in $\mathbf{C}_{n}(1)$. Thus $\mathbf{y} \in B(\mathbf{x}, 1)$, but is not in any other unit ball $B(\mathbf{z}, 1)$ with $\mathbf{z} \in \mathbf{C}_{n}(1)$.

Note that if $\mathbf{x} \in \mathbb{Z}_{3}^{n}$ is a perfect state, then applying one legal move to the $\mathbf{x}$ configuration may lead to two possible outcomes depending on where the smallest disk is transferred. Thus $B(\mathbf{x}, 1)$ has three elements. If $\mathbf{x} \in \mathbb{Z}_{3}^{n}$ is not a perfect state, then there are 3 possible outcomes with a legal move, namely, one may transfer the smallest disk to one of the two other pegs, or one may transfer a disk between the two pegs not containing the smallest disk. In such case, $B(\mathbf{x}, 1)$ has four elements. Now, the collection of $B(\mathbf{x}, 1)$ with $\mathbf{x} \in \mathbf{C}_{n}(1)$ form a partition of $\mathbb{Z}_{3}^{n}$. If $n$ is even, then all 3 perfect states belong to $\mathbf{C}_{n}(1)$. Hence 3 of the $B(\mathbf{x}, 1)$ will have 3 elements, and the rest will have 4 elements. Thus

$$
3^{n}=\left|\mathbb{Z}_{3}^{n}\right|=\sum_{\mathbf{x} \in \mathbf{C}_{n}(1)}|B(\mathbf{x}, 1)|=3 \times 3+4 \times\left(\left|\mathbf{C}_{n}(1)\right|-3\right),
$$

and thus $\left|\mathbf{C}_{n}(1)\right|=\left(3^{n}+3\right) / 4$. If $n$ is odd, there is only one perfect state in $\mathbf{C}_{n}(1)$. By a similar argument, one sees that $\left|\mathbf{C}_{n}(1)\right|=\left(3^{n}+1\right) / 4$.

We have several remarks in connection with the above theorem. Details and proofs can be found in [2].

1. The above proof actually suggests an easy decoding algorithm for $\mathbf{C}_{n}(1)$.

2. It is not difficult to show that one of the perfect states must be a codeword for any 1-error correcting code on $H_{n}$. If one assumes that the top corner vertex $0 \cdots 0$ is a codeword, then the code must be $\mathbf{C}_{n}(1)$.

3. By the general theory of coding theorem (for example, [4, Theorem 1.9]), we see that $d(\mathbf{x}, \mathbf{y}) \geqslant 3$ for any $\mathbf{x}, \mathbf{y} \in \mathbf{C}_{n}(1)$. This fact can also be proved independently using arguments similar to those in our proof of the theorem.

Next, we turn to the case when $k>1$. We have the following result.

THEOREM 2.2. Suppose $n, k>1$ are integers. There exists a perfect $k$-error correcting code $\mathrm{C}_{n}(k)$ on $H_{n}$ if and only if

(a) $k \geqslant 2^{n-2} \cdot 3$, or

(b) $k=2^{n-1}-1$.

Furthermore, if (a) holds, then a perfect $k$-error correcting code must consist of a single vertex $\mathrm{c}$ of $H_{n}$ such that $B(\mathrm{c}, k)$ contains all perfect states; if (b) holds, the only perfect $k$-error correcting code is the set of the three perfect states. 
As we shall see in the following proofs, it is rather easy to check that if $n$ and $k$ satisfy the condition (a) or (b), then there are perfect $k$-error-correcting codes $\mathbf{C}_{n}(k)$ as described in the theorem. The non-trivial part is the necessity part of the theorem, that is, the non-existence of any other perfect codes for $k>1$.

If $n>1$, we always assume that $H_{n}$ can be decomposed into $H_{n-1}^{(i)}$ for $i=0,1,2$, as described in the introduction.

We first establish several lemmas concerning the vertices of $H_{n}$.

LEMMA 2.3. Let $\mathrm{w}$ be a vertex in $H_{n}$, and let $d_{0}, d_{1}, d_{2}$ be the distance between $\mathrm{w}$ and the top, left bottom, and right bottom perfect states of $H_{n}$, respectively. Then

(a) $d_{0}, d_{1}, d_{2}$ cannot have the same (even or odd) parity.

(b) . $d_{i}=d_{j}$ for some $i \neq j$ if and only if $d_{3-i-j}=0$, that is, $w$ is a perfect state.

ProOF: The proof can be done by induction. If $n=1$, the conclusions (a) and (b) clearly hold. Suppose $n \geqslant 2$, and the conclusions hold for $H_{n-1}$. Let $\mathbf{w}$ be a vertex of $H_{n}$. We may assume that $\mathbf{w}$ lies in the subgraph $H_{n-1}^{(0)}$ of $H_{n}$ by a suitable 120 degrees rotation of $H_{n}$. Suppose $\mathbf{x}, \mathbf{y}, \mathbf{z}$ are the top, left bottom, and right bottom perfect states of $H_{n}$, and let $\tilde{\mathbf{y}}, \tilde{\mathbf{z}}$ be the left and right bottom perfect states of $H_{n-1}^{(0)}$ (see Figure 4). Then $d_{0}=d(\mathbf{w}, \mathbf{x})$,

$$
\begin{aligned}
& d_{1}=d(\mathbf{w}, \mathbf{y})=d(\mathbf{w}, \tilde{\mathbf{y}})+d(\tilde{\mathbf{y}}, \mathbf{y})=d(\mathbf{w}, \tilde{\mathbf{y}})+2^{n-1} \\
& d_{2}=d(\mathbf{w}, \mathbf{z})=d(\mathbf{w}, \tilde{\mathbf{z}})+d(\tilde{\mathbf{z}}, \mathbf{z})=d(\mathbf{w}, \tilde{\mathbf{z}})+2^{n-1}
\end{aligned}
$$

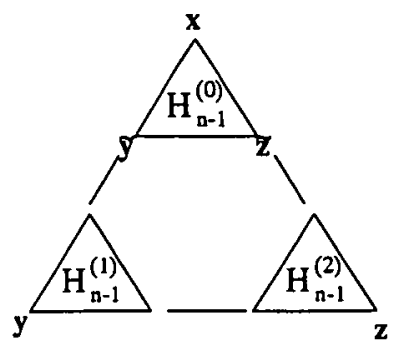

Figure 4

One can then apply the induction assumption on $d(\mathbf{w}, \mathbf{x}), d(\mathbf{w}, \tilde{\mathbf{y}})$ and $d(\mathbf{w}, \tilde{\mathbf{z}})$ to get the conclusions on $d_{0}, d_{1}$ and $d_{2}$.

Lemma 2.4. Let $n, k$ be positive integers. Suppose $\mathrm{w}$ is a vertex in $H_{n}$ such that $B(\mathbf{w}, k)$ does not contain any of the three perfect states. Then there is a subgraph $R$ of $H_{n}$ which is isomorphic to $H_{1}$ such that $B(\mathrm{w}, k)$ contains exactly one vertex of $R$.

Proof: Consider subgraphs in $H_{n}$ of the form $H_{m}$ so that the vertex set $V$ of the subgraph satisfies $\mathbf{w} \in V \subseteq B(\mathbf{w}, k)$. Let $S$ be such a subgraph with the maximum number of vertices, that is, largest possible $m$, and let $\mathbf{x}, \mathbf{y}, \mathbf{z}$ be the perfect states of $S$. Since $B(\mathbf{w}, k)$ does not contain any perfect state of $H_{n}$, we see that each of $\mathbf{x}, \mathbf{y}$ and $\mathbf{z}$ is connected to some vertices that are not in $S$ (see Figure 5). By Lemma 2.3 (a), we 
see that $n_{0}=k-d(\mathbf{w}, \mathbf{x}), n_{1}=k-d(\mathbf{w}, \mathbf{y})$ and $n_{2}=k-d(\mathbf{w}, \mathbf{z})$ cannot all have the same parity. In particular, we may assume that $n_{0}$ is odd. (Otherwise, apply 120 degrees rotations to $H_{n}$ to make $n_{0}$ odd.) Let $\mathbf{v}$ be a vertex in $H_{n}$ satisfying $d(\mathbf{w}, \mathbf{v})=k$ and $d(\mathbf{x}, \mathbf{v})=n_{0}$, and let $R$ be the subgraph of $H_{n}$, which is isomorphic to $H_{1}$ and has $\mathbf{v}$ as a vertex. If one follows a path in $H_{n}$ from $\mathbf{w}$ to $\mathbf{v}$, one sees that the two other vertices of the subgraph $R$ will have a distance $k+1$ from $\mathbf{w}$. Hence $B(\mathbf{w}, k)$ contains only the vertex $\mathbf{v}$ of $R$.

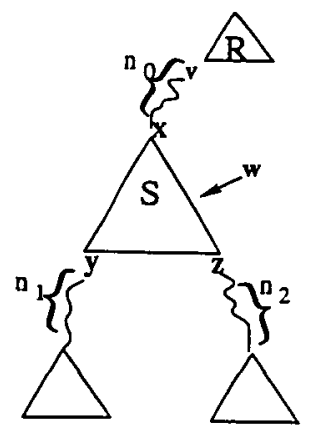

Figure 5

We are now ready to prove our theorem. While the theorem is stated in terms of the conditions on $n$ and $k$, we divide the proof into different cases according to the size of $\mathbf{C}_{n}(k)$. In particular, we shall show that $\left|\mathbf{C}_{n}(k)\right|$ can only be 1 or 3 . We begin with the case when $\left|\mathbf{C}_{n}(k)\right|=1$.

Lemma 2.5. Suppose $n, k>1$ are positive integers. Then there exists a perfect $k$-error correcting code on $H_{n}$ consisting of one codeword if and only if $k \geqslant 2^{n-2} \cdot 3$.

Proof: Let $\mathbf{x}, \mathbf{y}, \mathbf{z}$ be the top, left and right bottom perfect states of $H_{n}$, and let $\tilde{\mathbf{y}}, \tilde{\mathbf{z}}$ be the left and right bottom perfect states of $H_{n-1}^{(0)}$ (see Figure 4).

If $k \geqslant 2^{n-2} \cdot 3$, then one can choose $\mathbf{c}$ so that $d(\mathbf{c}, \widetilde{\mathbf{y}})=2^{n-2}$ and $d(\mathbf{c}, \widetilde{\mathbf{z}})=2^{n-2}-1$. One easily checks that all vertices of $H_{n}$ lie in $B(\mathrm{c}, k)$.

Conversely, suppose there is a perfect $k$-error correcting code consisting of the single codeword c. Then all perfect states belong to $B(\mathbf{c}, k)$. To show that $k \geqslant 2^{n-2} \cdot 3$, suppose c lies in $H_{n-1}^{(0)}$, otherwise apply a suitable 120 degrees rotation to $H_{n}$. Then

$$
2^{n-1}-1=d(\tilde{\mathbf{z}}, \tilde{\mathbf{y}}) \leqslant d(\tilde{\mathbf{z}}, \mathbf{c})+d(\mathbf{c}, \tilde{\mathbf{y}}) .
$$

It follows that

$$
\begin{aligned}
k & =\max \{d(\mathbf{c}, \mathbf{z}), d(\mathbf{c}, \mathbf{y})\} \\
& =\max \{d(\mathbf{c}, \tilde{\mathbf{z}})+d(\mathbf{z}, \tilde{\mathbf{z}}), d(\mathbf{c}, \tilde{\mathbf{y}})+d(\mathbf{y}, \tilde{\mathbf{y}})\} \\
& =\max \{d(\mathbf{c}, \tilde{\mathbf{z}}), d(\mathbf{c}, \tilde{\mathbf{y}})\}+2^{n-1} \\
& \geqslant\left[2^{n-1} \cdot 3-1\right] / 2
\end{aligned}
$$


Since $k$ is an integer, we have $k \geqslant 2^{n-2} \cdot 3$.

Next, we consider the case when $1<\left|\mathbf{C}_{n}(k)\right| \leqslant 3$.

LEMma 2.6. Suppose $n, k>1$ are positive integers. The following conditions are equivalent.

(a) There exists a perfect $k$-error correcting code $\mathrm{C}_{n}(k)$ on $H_{n}$ with $1<$ $\left|\mathbf{C}_{n}(k)\right| \leqslant 3$.

(b) There is a unique perfect $k$-error correcting code on $H_{n}$ consisting of the 3 perfect states.

(c) $k=2^{n-1}-1$.

Proof: It is clear that (b) implies (a). If (c) holds, then the perfect states will form a perfect $k$-error correcting code, and thus (a) is true. It remains to prove that both (b) and (c) follow from (a).

Now, suppose (a) holds. Let $\mathbf{x}, \widetilde{\mathbf{y}}, \tilde{\mathbf{z}}$ be the top, left and right bottom perfect states of $H_{n-1}^{(0)}$ (see Figure 4).

We first show that it is impossible to have $\left|\mathbf{C}_{n}(k)\right|=2$. Suppose there is such a perfect code, and $\mathbf{C}_{n}(k)$ consists of $\mathbf{c}_{1}$ and $\mathbf{c}_{2}$. We may assume that they are not vertices of $H_{n-1}^{(0)}$ by a suitable 120 degrees rotation of $H_{n}$. Then $\mathrm{x}$ lies in one of two radius- $k$ balls centred at the codewords. Without loss of generality, we may assume that $\mathbf{x}$ lies in $B\left(\mathbf{c}_{1}, k\right)$ and $\mathbf{c}_{\mathbf{l}}$ is a vertex of $H_{n-1}^{(1)}$. Then $k \geqslant d\left(\mathbf{c}_{1}, \mathbf{x}\right)>d(\tilde{\mathbf{y}}, \mathbf{x})$, or in other words, $k \geqslant 2^{n-1}$ Clearly, $\mathbf{c}_{2}$ cannot lie in $H_{n-1}^{(1)}$; otherwise, $\tilde{\mathbf{y}} \in B\left(\mathbf{c}_{1}, k\right) \cap B\left(\mathbf{c}_{2}, k\right)$ by the above condition on $k$. Also, $\mathrm{c}_{2}$ cannot lie in $H_{n-1}^{(2)}$, otherwise, $\tilde{\mathbf{z}} \in B\left(\mathrm{c}_{1}, k\right) \cap B\left(\mathrm{c}_{2}, k\right)$. Thus $\mathrm{C}_{n}(k)$ cannot have exactly 2 elements.

Next, suppose $\mathbf{C}_{n}(k)$ consists of 3 elements: $\mathbf{c}_{0}, \mathbf{c}_{1}, \mathbf{c}_{2}$. Notice that each of $H_{n-1}^{(i)}$ for $i=0,1,2$, has a codeword; that is, $\mathbf{c}_{i}$ is a vertex of $H_{n-1}^{(i)}$. If this is not the case, one can use the arguments in the preceding paragraph to show that two of the $B\left(\mathbf{c}_{i}, k\right)$ will have non-empty intersection. If $B\left(\mathbf{c}_{i}, k\right)$ only contains those vertices in $H_{n-1}^{(i)}$ for $i=0,1,2$, then we have $d\left(\mathbf{c}_{0}, \tilde{\mathbf{y}}\right)=d\left(\mathbf{c}_{0}, \widetilde{\mathbf{z}}\right)$. By Lemma $2.3(\mathrm{~b})$, we see that $\mathbf{c}_{0}=\mathbf{x}$ and $k=2^{n-1}-1$. Similarly, one can show that $\mathbf{c}_{1}=\mathbf{y}$ and $\mathbf{c}_{2}=\mathbf{z}$. Hence condition (b) and (c) of the lemma hold.

In the following, we show that any other construction of $\mathbf{C}_{n}(k)$ is impossible. Suppose it is not the case, and suppose one of the $B\left(c_{i}, k\right)$ contains some vertices in $H_{n-1}^{(j)}$ for some $i \neq j$. We may assume that $(i, j)=(0,2)$ by applying some suitable rotation and reflection about the vertical line passing through $\mathbf{x}$. Furthermore, decompose $H_{n-1}^{(2)}$ into $H_{n-2}^{(0)}, H_{n-2}^{(1)}$, and $H_{n-2}^{(2)}$ as shown in Figure 6. 


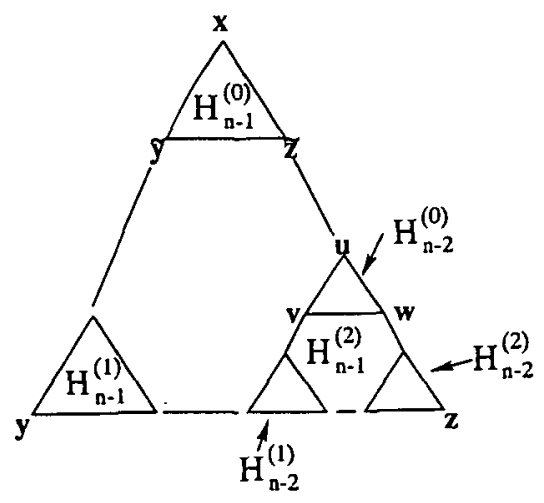

Figure 6

Suppose $\mathbf{u}, \mathbf{v}$ and $\mathbf{w}$ are the top, left and right bottom perfect states of $H_{n-2}^{(0)}$. Then $\mathbf{u} \in B\left(\mathbf{c}_{0}, k\right)$ by our assumption. Note that

$$
d\left(\mathbf{c}_{0}, \mathbf{v}\right)=d\left(\mathbf{c}_{0}, \tilde{\mathbf{z}}\right)+d(\tilde{\mathbf{z}}, \mathbf{v})=d\left(\mathbf{c}_{0}, \widetilde{\mathbf{z}}\right)+d(\widetilde{\mathbf{z}}, \mathbf{w})=d\left(\mathbf{c}_{0}, \mathbf{w}\right)
$$

Thus either

(i) $\mathbf{v}, \mathbf{w} \in B\left(\mathrm{c}_{0}, k\right)$, or

(ii) $\{\mathbf{v}, \mathbf{w}\} \cap B\left(\mathbf{c}_{0}, k\right)=\emptyset$.

We first show that (i) cannot happen. If $\mathbf{v}, \mathbf{w} \in B\left(\mathbf{c}_{0}, k\right)$, then $k \geqslant d\left(\mathbf{c}_{0}, \mathbf{w}\right)$ $\geqslant d(\widetilde{\mathbf{z}}, \mathbf{w})=2^{n-2}$, and we must have $\mathbf{c}_{2}$ lying in $H_{n-2}^{(1)}$ or $H_{n-2}^{(2)}$. If $\mathbf{c}_{2}$ is a vertex of $H_{n-2}^{(1)}$, then $\mathbf{v} \in B\left(\mathbf{c}_{2}, k\right)$ as $k \geqslant 2^{n-2}$. If $\mathbf{c}_{2}$ is a vertex of $H_{n-2}^{(2)}$, then $\mathbf{w} \in B\left(\mathrm{c}_{2}, k\right)$ as $k \geqslant 2^{n-2}$. In both cases, we have $B\left(\mathbf{c}_{0}, k\right) \cap B\left(\mathbf{c}_{2}, k\right) \neq \emptyset$, which is a contradiction.

Next, we show that (ii) is also impossible. Note that by an argument similar to that in the preceding paragraph, one can show that even if $B\left(c_{1}, k\right)$ contains some vertices in $H_{n-1}^{(2)}$, it cannot include all the vertices in $H_{n-2}^{(1)}$. Thus $B\left(c_{1}, k\right)$ cannot contain $\mathbf{v}$ or $\mathbf{w}$, and so we must have $\mathbf{v}, \mathbf{w} \in B\left(\mathbf{c}_{2}, k\right)$. If $\mathbf{c}_{2}$ is a vertex in $H_{n-2}^{(0)}$, then $k<2^{n-2}$. Otherwise, we have $d\left(\mathbf{c}_{2}, \mathbf{u}\right) \leqslant 2^{n-2} \leqslant k$ and thus $\mathbf{u} \in B\left(\mathbf{c}_{2}, k\right) \cap B\left(\mathbf{c}_{0}, k\right)$. But then $k<2^{n-2} \leqslant d\left(\mathbf{c}_{i}, \mathbf{z}\right)$ for any $i$, contradicting the fact that $\mathbb{Z}_{3}^{n}=\bigcup_{i=0}^{2} B\left(\mathbf{c}_{i}, k\right)$. Thus, $\mathbf{c}_{2}$ must be in either $H_{n-2}^{(1)}$ or $H_{n-2}^{(2)}$. Now consider the vertex $\tilde{\mathbf{w}}$ on the shortest path from $\mathbf{u}$ to $\mathbf{w}$ such that $d\left(\mathbf{c}_{0}, \widetilde{\mathbf{w}}\right)=k+1$. Clearly, we have

$$
k=d\left(\mathbf{c}_{2}, \tilde{\mathbf{w}}\right)=d\left(\mathbf{c}_{2}, \mathbf{w}\right)+d(\mathbf{w}, \tilde{\mathbf{w}})
$$

Similarly, if $\tilde{\mathbf{v}}$ is the vertex on the shortest path from $\mathbf{u}$ to $\mathbf{v}$ such that $d\left(\mathbf{c}_{0}, \widetilde{\mathbf{v}}\right)=k+1$, then

$$
k=d\left(\mathbf{c}_{2}, \tilde{\mathbf{v}}\right)=d\left(\mathbf{c}_{2}, \mathbf{v}\right)+d(\mathbf{v}, \tilde{\mathbf{v}}) .
$$

Evidently, $d(\mathbf{u}, \tilde{\mathbf{w}})=d(\mathbf{u}, \tilde{\mathbf{v}})=(k+1)-d\left(\mathbf{c}_{0}, \mathbf{u}\right)$. It follows that $d(\mathbf{w}, \tilde{\mathbf{w}})=d(\mathbf{v}, \tilde{\mathbf{v}})$ and $d\left(\mathbf{c}_{2}, \mathbf{v}\right)=d\left(\mathbf{c}_{2}, \mathbf{w}\right)$. However, this is impossible because $d\left(\mathbf{c}_{2}, \mathbf{v}\right) \leqslant 2^{n-2}<d\left(\mathbf{c}_{2}, \mathbf{w}\right)$ if $\mathbf{c}_{2} \in H_{n-2}^{(1)}$, and $d\left(\mathbf{c}_{2}, \mathbf{w}\right) \leqslant 2^{n-2}<d\left(\mathbf{c}_{2}, \mathbf{v}\right)$ if $\mathbf{c}_{2} \in H_{n-2}^{(2)}$. 
Finally, we consider the case when $3<\left|\mathbf{C}_{n}(k)\right|$.

Lemma 2.7. Suppose $n, k>1$ are positive integers. Then there is no perfect $k$-error correcting code $\mathbf{C}_{n}(k)$ on $H_{n}$ with $3<\left|\mathbf{C}_{n}(k)\right|$.

Proof: Suppose there is a perfect $k$-error correcting code $\mathrm{C}_{n}(k)$ on $H_{n}$ with $3<$ $\left|\mathbf{C}_{n}(k)\right|$. Then there is a codeword $\mathbf{c}$ such that $B(\mathbf{c}, k)$ does not contain any of the three perfect states of $H_{n}$. By Lemma 2.4, there exists a subgraph $U$ of $H_{n}$ with vertices $\mathbf{u}_{0}, \mathbf{u}_{1}$ and $\mathbf{u}_{2}$ isomorphic to $H_{1}$ such that $B(\mathbf{c}, k) \cap U=\mathbf{u}_{0}$. Furthermore, we assume that $\mathbf{u}_{\mathbf{1}}$ is connected to a subgraph $V$ with vertices $\mathbf{v}_{\mathbf{0}}, \mathbf{v}_{\mathbf{1}}, \mathbf{v}_{\mathbf{2}}$, which is also isomorphic to $H_{1}$. Similarly, $\mathbf{u}_{2}$ is connected to a subgraph $W$ with vertices $\mathbf{w}_{0}, \mathbf{w}_{1}, \mathbf{w}_{2}$, which is also isomorphic to $H_{1}$. We depict the situation in Figure 7.

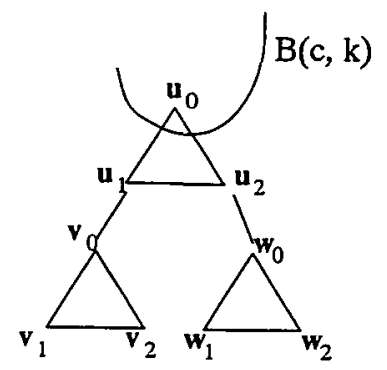

Figure 7

Let $\mathrm{d}$ be a codeword such that $\mathbf{u}_{1} \in B(\mathrm{~d}, k)$. Since $k \geqslant 2$, we can move at least 2 steps from $\mathbf{u}_{1}$ along the shortest path from $\mathbf{u}_{1}$ to $\mathbf{d}$ in $H_{n}$. Clearly, $d\left(\mathbf{d}, \mathbf{u}_{1}\right)=k$ and hence $d\left(\mathbf{d}, \mathbf{v}_{0}\right)=k-1$ so that $B(\mathbf{c}, k) \cap B(\mathbf{d}, k)=\emptyset$. As a result, $d\left(\mathbf{d}, \mathbf{v}_{i}\right)=k-2$ for $i=1$ or 2 . Similarly, let $\mathbf{e}$ be a codeword such that $\mathbf{u}_{2} \in B(\mathbf{e}, k)$. One then sees that $d\left(\mathbf{e}, \mathbf{w}_{0}\right)=k-1$, and $d\left(\mathbf{e}, \mathbf{w}_{\mathbf{i}}\right)=k-2$ for $i=1$ or 2 .

We consider 2 cases. First, suppose $\mathbf{v}_{2}$ and $\mathbf{w}_{1}$ are adjacent. Since $d\left(\mathbf{v}_{1}, \mathbf{w}_{1}\right)=2$ and $d\left(\mathbf{v}_{2}, \mathbf{w}_{1}\right)=1$, we see that

$$
d\left(\mathbf{w}_{1}, \mathbf{d}\right) \leqslant \min \left\{d\left(\mathbf{w}_{1}, \mathbf{v}_{i}\right)+d\left(\mathbf{v}_{i}, \mathbf{d}\right): i=1,2\right\} \leqslant k .
$$

Thus $\mathbf{w}_{1} \in B(\mathbf{d}, k) \cap B(\mathbf{e}, k)$, which is a contradiction unless $\mathbf{d}=\mathbf{e}$. However, this is impossible as shown in the following. If $k=2$, then $d$ must be chosen from $\left\{\mathbf{v}_{1}, \mathbf{v}_{2}, \mathbf{w}_{1}, \mathbf{w}_{2}\right\}$. But none of the choices will lead to $d\left(\mathbf{d}, \mathbf{v}_{0}\right)=k-1=d\left(\mathbf{d}, \mathbf{w}_{0}\right)$. Thus we may assume that $k>2$, and $d\left(\mathbf{d}, \mathbf{v}_{1}\right)=k-2=d\left(\mathbf{d}, \mathbf{w}_{2}\right)$. But then $\mathbf{d}$ must lie in some subgraph $R$ isomorphic to $H_{r}$ so that the vertex set of $R$ is contained in $B(\mathrm{~d}, k-3)$. If one moves $k$ steps along a path from $\mathbf{d}$ to the subgraph $U$, either one can reach exactly one vertex of $U$ or all the three vertices of $U$ (see Proof of Lemma 2.4). Thus, it is impossible to have $\mathbf{u}_{1}, \mathbf{u}_{2} \in B(\mathrm{~d}, k)$.

Next, suppose $\mathbf{v}_{2}$ and $\mathbf{w}_{1}$ are not adjacent. Then $U$ must be lying at the bottom of a certain subgraph $S$ of $H_{n}$ which is isomorphic to some $H_{m}$ with $m>1$, and either

(i) both $V$ and $W$ are in the same $H_{m}$, or 
(ii) only one of $V$ or $W$ is also in $H_{m}$.

In both cases, there will be a subgraph $\widetilde{S}$ of $S$ isomorphic to $H_{2}$ containing $U$ and one of $V$ or $W$, say $W$ (see Figure 8). Since both $\mathrm{u}_{0}$ and $\mathrm{w}_{2}$ have a distance 2 from the top perfect state of $\widetilde{S}$, which has a distance $k-2$ from $\mathbf{c}$, we see that $\mathbf{w}_{2} \in B(\mathbf{c}, k)$. However, $d\left(\mathbf{e}, \mathbf{w}_{0}\right)=k-1$ implies that $\mathbf{w}_{2}$ also belongs to $B(\mathbf{e}, k)$, which is a contradiction.

One can easily combine Lemmas $2.5-2.7$ to get the conclusion of Theorem 2.2.

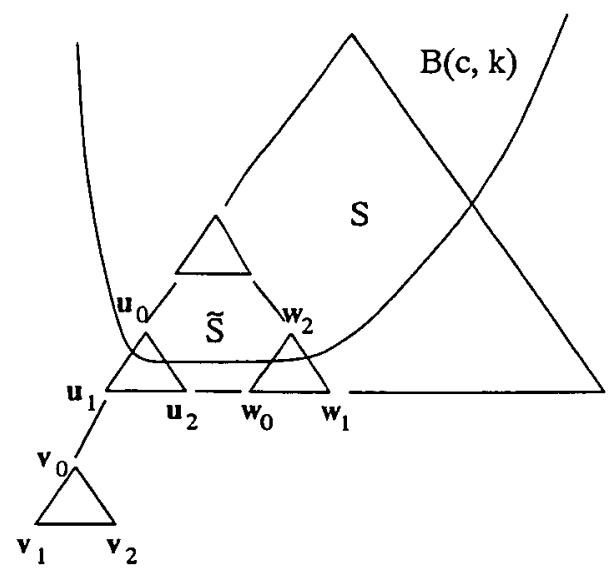

Figure 8

\section{REFERENCES}

[1] R.A. Brualdi, Introductory combinatorics (North-Holland, New York, 1992).

[2] P. Cull and I. Nelson, 'Error-correcting codes on the Towers of Hanoi graphs', (preprint).

[3] M.C. Er, 'The cyclic towers of Hanoi and pseudo ternary codes', J. Inform. Optim. Sci. 6 (1986), 271-277.

[4] R. Hill, A first course in coding theory (Oxford University Press, Oxford, 1986).

[5] A.M. Hinz, 'The Tower of Hanoi', Enseign. Math. 35 (1989), 289-321.

[6] R.S. Scorer, P.M. Grundy and C.A.B. Smith, 'Some binary games', Math. Gaz. 280 (1944), 96-103.

Department of Mathematics

The College of William and Mary

Williamsburg, VA 23187

United States of America

e-mail: ckli@math.wm.edu
Lockheed Martin Federal Systems

6304 Spine Road

Boulder CO 80302

United States of America

e-mail: ingrid.nelson@lmco.com 